\title{
Knowledge of Mothers about Side Effects of Obligatory Vaccines in Al-amyria Family Medicine Center, Cairo
}

\author{
*Mohamed Y. El Awady, *Nahla F. Abo El Ezz, *Mohamed T. Momen, \\ **Mahmoud M. Metwaly
}

*Community, Environmental and Occupational Medicine department, faculty of medicine, Ain Shams University

**Family Physician in Cairo Health insurance chicer

Received: July 2015, accepted: October2015

\begin{abstract}
:
Knowledge of mothers about vaccination has been documented to influence the decisions they make when it comes to vaccinating their children. The aim of this study was to measure the prevalence of mothers' knowledge about vaccination side effects and determine any significant relationships between the mothers' demographic characters and their knowledge. Methods: The mothers were also asked about their source of information. The study was carried out at Al-Amyria Family Centre in Cairo and included a total of 385 mothers who were there for their child's vaccination session. Results: The prevalence of mothers who had knowledge about side effects was $78.7 \%$, with $32.5 \%$ responding that a healthcare provider was the main source, and $26.5 \%$ responding that television was the main source of information. These two sources were the main ones, regardless of the mothers' age or education. Nearly all mothers $(98.7 \%)$ said they continue giving booster doses to their children even if side effects occur. Conclusion: Programs to enhance the mothers' knowledge and provide more information to them can therefore be developed with healthcare professionals playing a major role.
\end{abstract}

Keywords: vaccines, mothers, side effects, knowledge

*corresponding author, Mahmoud M. Metwaly Email: mmetwaly6@hotmail.com

\section{Introduction}

Vaccination is a proven and one of the most cost effective child survival interventions. All countries in the world have an immunization programme to deliver selected vaccines to the targeted beneficiaries, specially focusing on pregnant women, infants and children, who are at a high risk of diseases preventable by vaccines. There are at least 27 causative agents against which vaccines are available and many more agents are targeted for development of vaccines. The number of antigens in the immunization programmes varies from country to country; however, there are a few selected antigens against diphtheria, pertussis, tetanus, poliomyelitis, measles, hepatitis B which are part of immunization programmes in most of the countries in the world ${ }^{(1)}$.

Regarding Bacille Calmette-Guérin (BCG), an attenuated vaccine derived from Mycobacterium bovis, is the current vaccine of choice against tuberculosis (TB). Despite its protection against activeTB in children, BCG has failed to protect adults againstTB infection and active disease development, especially in developing countries where the disease is endemic. Currently, there is a significant effort toward the development of a new TB vaccine ${ }^{(2)}$. 
The Measles-Mumps-Rubella (MMR) vaccine is generally administered to children around the age of one year, with a second dose before starting school (i.e. age 4-5years). The second dose is a dose to produce immunity in the small number of persons $(2-5 \%)$ who fail to develop measles immunity after the first dose.

The MMRV vaccine, a combined measles, mumps, rubella and varicella vaccine, has been proposed as a replacement for the MMR vaccine to simplify administration of the vaccines (3).

The most common minor side effects from a polio vaccination include irritability up to $64.5 \%$, tiredness up to $60.7 \%$, tenderness at the injection site up to $29.4 \%$, loss of appetite up to $16.6 \%$, persistent crying up to $1.4 \%$ and redness at the injection site up to $1 \%{ }^{(4)}$.

Side effects from hepatitis B vaccine are uncommon and usually mild, but may include localised pain, redness and swelling at the injection site occasionally, an injection-site lump (nodule) that may last many weeks, but treatment is not needed low-grade temperature (fever) children who can be unsettled, irritable, cry, are generally unhappy, drowsy and tired (5).

\section{Aim of the Study}

To measure the knowledge of mothers about common and potential sideeffects of obligatory vaccines (BCG, DPT, polio vaccine, hepatitis B vaccine, MMR)

To identify frequency of different side effects i.e. fever, indurations at site of injection, pain.

\section{Subjects and Methods}

Study design: A cross sectional study was conducted to determine the knowledge of mothers about side effects of obligatory vaccines in AlAmyria family medicine Center- Cairo

Study population: Mothers attending vaccinations sessions in Al-Amyria Family Medicine Center, the study started in December 2014 and continued till April 2015.

Study Sample: Subjects in the study were recruited over 3 days per week on alternative manner during the complete vaccination sessions of the day.

Study sample size: A sample of 385 mothers were interviewed, the sample was calculated using Epi-info 7.1 program putting in consideration prevalence of knowledge about adverse effects of vaccines $66 \%$ (6) at confidence interval $95 \%$.

\section{The study tool:}

An interview questionnaire that included questions on:

\section{A- Socio demographic} characteristics:

Age of mothers, infant age, educational status of mothers, her work, number of children she has, education and job of the father.

\section{B- Knowledge of mothers about:}

1- Importance of vaccination for the child.

2- The source of knowledge about vaccination importance.

3- The knowledge of mothers about the side effects of vaccination.

4- The source of knowledge about the side affect of vaccination

5- What are the side effects of vaccination that happened for the child?

6- In case of occurrence of side effect was the vaccination is completed?

\section{Data management and analysis}

All data were coded and kept confidential.

Data collected from 385 mothers. 
The collected data was revised, coded, tabulated and introduced to aPC using statistical package for social science (SPSS 15.0.1 for windows, SPSS Inc, Chicago, IL, 2001).

\section{Ethical Consideration:}

Participants were interviewed after taking their verbal consent. Administrative approval obtained from Al-Amyria Family Medicine Center.

\section{Results}

Table (1) shows that $(78.7 \%)$ of the studied mothers had knowledge about the side effects of vaccination.

Table (2) shows that $(70.9 \%)$ of the studied mothers reported that their infants had fever, (19.5\%) had indurations and $(8.6 \%)$ had pain as a side effect following vaccination.

Table (3) shows that $98.7 \%$ of the studied mothers continue giving booster doses to their children in case of side effects occurrence in 1st dose.

\section{Discussion}

Maternal knowledge as well as maternal educational level have been documented to influence immunization uptake $^{(6)}$.

Parental decision regarding immunization is very important for increasing the immunization rate and compliance and for decreasing any possible immunization errors. Parents' knowledge and practices regarding immunization are the major factors that contribute to their vaccination decision (7)

This study aimed to measure the knowledge of mothers about common and potential side effects of obligatory vaccines (BCG, DPT, polio vaccine, hepatitis $\mathrm{B}$ vaccine, MMR vaccine) and to identify the frequency of different side effects following vaccination as fever, induration at site of vaccination and pain. In order to achieve the study objectives, a cross sectional study design was conducted in which a sample of 385 mothers attending vaccination sessions in AlAmariya Family Medicine CentreCairo, were interviewed.

The percentage of mothers in the current study, who obtained their knowledge about vaccination importance from health care providers, is rather lower than that found in a study conducted in the United Arab Emirates ${ }^{(8)}$, which found that about $50 \%$ of the subjects obtained information on immunization importance from the health professionals.

This was supported by a study by done in Pakistan ${ }^{(9)}$, which also identified that the health care staff was the main source of information about immunization. This difference between our study and the other studies may be due to cultural difference, as the mothers in Egypt rely on other sources of information like the television and family members.

Results of the current study show that $78.7 \%$ of the studied mothers had knowledge about side effects of vaccination; while in another study ${ }^{(10)}$ $34.0 \%$ of the mothers could not mention any of the adverse events that may follow immunization. On the other hand, findings from a study in Nigeria (6) have concluded that although a good number of the participants heard about adverse events following immunization, there is a poor maternal detailed knowledge about adverse events of immunization in Enugu, Nigeria.

In the current study, the majority of the studied mother $(70.9 \%)$ reported that their infant had fever, $(19.5 \%)$ had induration at site of vaccine injection 
and $(8.6 \%)$ had pain as a side effect following vaccination. This is in consistence with the research done in Nigeria (11) which indicated that the majority of respondents $(63.1 \%)$ claimed that when they immunized their children, they observed some type of side effect as fever, rash, pain and swelling.

This study results show that fever was the main side effect occurring after vaccination in all infant ages; while another study ${ }^{(12)}$ stated that about $50 \%$ and more of the respondents reported that each vaccine (BCG, polio vaccine, DPT, measles) caused mild fever.

\section{References:}

1) Chandrakant Lahariya,(2014) ; A brief history of vaccines \& vaccination in India, Indian $\mathrm{J}$ Med Res 139,p 491-511.

2) Mangtani $P$, Beynon $R$ and Pimpin $L$ et al. (2014) : Protection by BCG vaccine against tuberculosis : asystemic review of randomized controlled trials. Clin Infect Dis - 58:470-80.

3) Vesikari $T$, Rentier $B$ and Gershon A (2010) : "Increasing coverage and efficiency of measles, mumps, and rubella vaccine and introducing universal varicella vaccination in Europe: a role for the combined vaccine". Pediatr Infect Dis J 26 (7): 632-8.

4) Plotkin SA, Orenstein $W$ and Offit PA. (2012) : Poliovirus vaccine-inactivated and poliovirus vaccine-live in Vaccines, 6th Edition, 573-645.

5) Shepard CW, Simard EP and Finelli L,et al ., (2010) : Hepatitis $B$ virus infection: epidemiology and vaccination. Epidemiol Rev 28: 112-125.

6) Nnenna, Uleanya and Omotowo, Mothers Knowledge and Pereption of Adverse events following immunization in Enugu, southEast, Nigeria, 2013.

7) Gellin $B$, Maibach $E$ and Marcuse E. Do parents understand immunizations? A national telephone survey. Pediatrics 200; 106 (5): 1097-1102.

8) Roos M., Fatma R. \& Noura A. Knowledge, attitude and practice towards immunizations among mothers in a traditional City in the united Arab Emirates, Journal of medical Sciences, 2011; 4 (3): 114-121.

9) Nisar, N., Mirza, M., \& Qadri, M. H. Knowledge, Attitude and Practices of mothers regarding immunization of one year old child at Mawatch Goth, Kemari Town, Karachi. Pak J Med Sci, 2010, 26(1), 183-186.

10) Shah B, Sharrna M, Vani $S$ $N$. Knowledge, altitude and practice of immunization in an urban educated population. Indian J Pediatr, 199158 (5): 691-695.

11) Shah B, Sharrna M, Vani $S$ $N$. Knowledge, altitude and practice of immunization in an urban educated population. Indian J Pediatr, 199158 (5): 691-695.

12) Muh-Asem. Malik $N$ and Yousaf $\boldsymbol{H}$. An assessment of parental knowledge. Belief and Attitude toward childhood immunization among minorities in Rural Areas of District Faisalabad. Pakistan. Mediterranean Journal of Social Sciences Vol. 3 (11) Nov 2012. 
Table (1): Frequency Distribution of the Studied Mothers According to Knowledge about Side Effects of Vaccination $(n=377)$

\begin{tabular}{|l|c|c|}
\hline Knowledge about Side Effects of Vaccination & No. & \% \\
\hline \hline No & 74 & 19.2 \\
\hline Yes & 303 & 78.7 \\
\hline Not Mentioned & 8 & 2.1 \\
\hline \hline Total & 377 & 100.0 \\
\hline
\end{tabular}

Table (2): Frequency Distribution of Reported Side Effects following Vaccination $(\mathbf{n}=\mathbf{3 8 1})$

\begin{tabular}{|l|c|c|}
\hline Side Effects of Vaccination & No. & \% \\
\hline \hline Fever & 273 & 70.9 \\
\hline Induration & 75 & 19.5 \\
\hline Pain & 33 & 8.6 \\
\hline Not Mentioned & 4 & 1.0 \\
\hline \hline Total & 385 & 100.0 \\
\hline
\end{tabular}

